\title{
Application of Computational Homology and Graph-Theoretic Approaches for Quantitative Chemical Imaging in Atom Probe Tomography
}

Scott Broderick ${ }^{1}$, Joaquin Peralta ${ }^{1}$, Sai Samudrala ${ }^{2}$, Kaustubh Kaluskar ${ }^{1}$, Baskar Ganapathysubramanian $^{2}$, Krishna Rajan ${ }^{1, *}$

${ }^{1}$ Department of Materials Science and Engineering, Iowa State University, Ames, IA 50011

${ }^{2}$ Department of Mechanical Engineering, Iowa State University, Ames, IA 50011

This presentation describes a methodology for quantitative characterization of precipitate morphology in atom probe tomography which accounts for both the statistical uncertainty associated with missing data as well as the physics of preferential evaporation. The procedure involves two steps. The first involves the use of data mining methodologies applied to the mass spectra to enhance the chemical resolution of the APT, including for discrimination of precipitate and matrix atoms [1]. The spectral resolution is enhanced by improved signal-to-noise and discrimination isotopes with overlapping mass-to-charge ratios $(\mathrm{m} / \mathrm{n})$. A non-parametric statistical analysis rapidly and robustly enhances the deconvolution of peaks in the mass spectrum to resolve isotopes (Figure 1). By accurately defining background, statistically reducing noise in the data, and addressing overlapping peaks, compositional measurements are improved and more reliable identification of atom species in the spatial image are achieved. The precipitate region is then defined by applying computational homology approaches to the point cloud data of the 3D APT image. We leverage powerful graph-based algorithms to identify the local topology of precipitates without the necessity of any heuristics [2] (Figure 2). The change in evaporation physics for the precipitate versus the matrix is defined based on the combination of first principles calculations and ion evaporation maps [3]. We link the data mining-enhanced measurement of precipitate chemistry and graph-based definition of precipitate topology with the first principles modeling of evaporation physics for enhanced APT chemical resolution [4].

\section{References}

[1] S. Broderick, A. Bryden, S. Suram, K. Rajan., Ultramicroscopy (In Press)

[2] S. Samudrala, O. Wodo, S. Suram, S. Broderick, K. Rajan, B. Ganapathysubraminian, Comp. Mat. Sci, (In Press)

[3] J. Peralta, S. Broderick, K. Rajan, Ultramicroscopy (In Press)

[4] Support comes from Air Force Office of Scientific Research grants: FA9550-10-1-0256, FA9550-11-1-0158 and FA9550-12-0496; NSF grants: ARI Program CMMI-09-389018 and PHY CDI-09-41576; and Defense Advanced Research Projects Agency grant N66001-10-14004. 

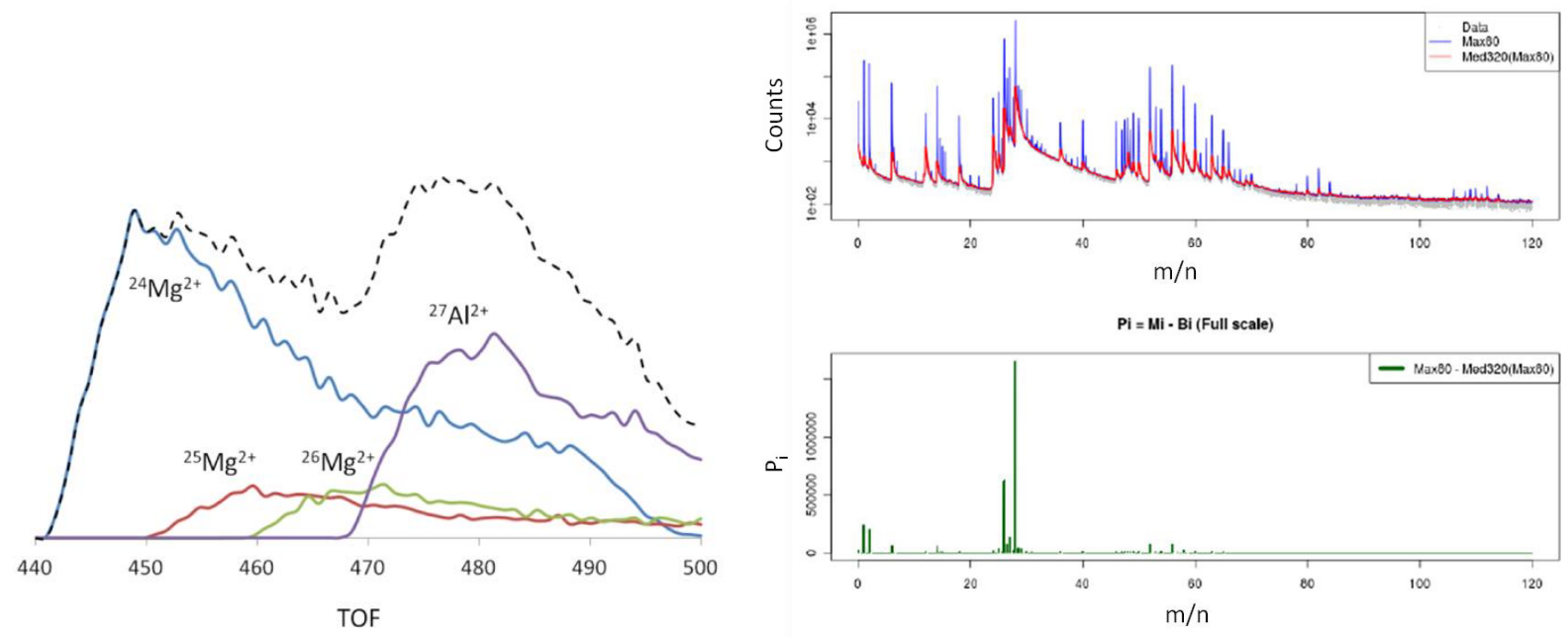

Figure 1. Improved chemical resolution of APT mass spectra. (a) A mass spectra where the isotopes are assigned chemical identity from data mining analysis and overlapping peaks are discriminated. (b) A mass spectra with background and noise removed, so that chemical signal is enhanced and the significant peaks are identified.
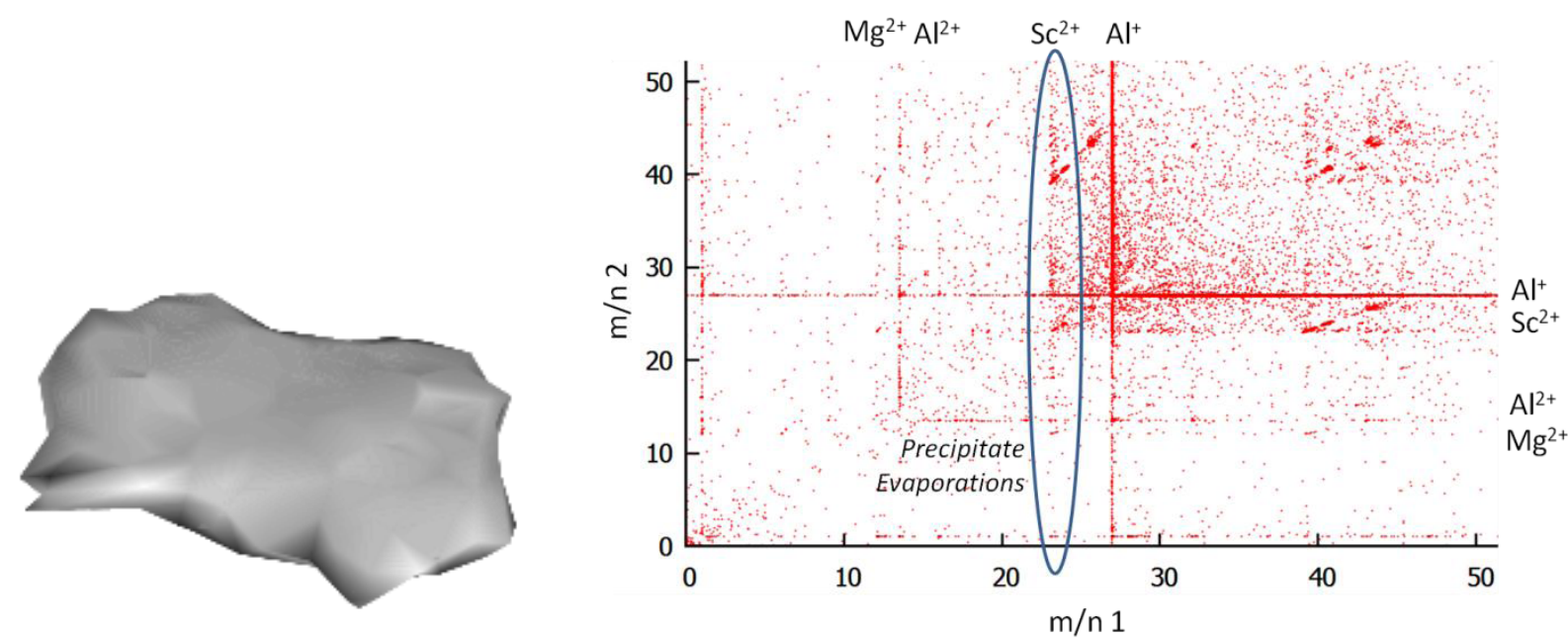

Figure 2. Quantitative definition of topology and evaporation physics for $\mathrm{Al}_{3} \mathrm{Sc}$ precipitate. (a) Non-convex surface of precipitate defined through graph-theoretic approach. The spatial analysis of APT is enhanced through quantitatively defined topology. (b) Ion evaporation map to link evaporation of precipitate ions with first principles calculations. 